\title{
La familia como factor decisorio en la elección del magisterio
}

\author{
Juan Luis Sánchez Villanueva ${ }^{1}$ \\ Doctor en Historia por la Universidad de Cádiz \\ juanluissanchez49@yahoo.es
}

RESUMEN: Aproximación a la situación de las mujeres en la legislación educativa del siglo XIX. La influencia de la familia en la elección del magisterio como actividad laboral. El Archivo de la Universidad de Cádiz como base de la documentación utilizada.

Palabras clave: Mujeres, Legislación educativa, Familia, Archivo de la Universidad de Cádiz.

\section{The family as a deciding factor in the choice of teaching}

ABSTRACT: Approach to the situation of women in the educational legislation of the $19^{\text {th }}$ century. The influence of the family in the choice of teaching as a work activity. The General Archive of the UCA as the basis of the documentation used.

Keywords: Women, Educational legislation, Family, Archivo de la Universidad de Cádiz.

Desde los primeros años del siglo XIX las leyes y normas relacionadas con la enseñanza han dejado clara la diferencia de trato entre niños y niñas. El Proyecto de decreto para el arreglo general de la enseñanza pública, de 7 de marzo de 1814, firmado entre otros por Manuel Josef de la Quintana y Josef de Vargas y Ponce, dice en su artículo 8 que «La primera enseñanza es la general é indispensable que debe darse á la infancia»; en su artículo 9 leemos, «Esta primera enseñanza se dará á los niños...»; y el artículo 10 añade «En estas escuelas aprenderán los niños á leer con sentido, y á escribir con claridad y buena ortografía é igualmente las reglas elementales de la aritmética, un catecismo religioso y moral, que comprenda brevemente los dogmas de la Religión y las máximas principales de buena conducta y buena crianza».

\footnotetext{
${ }^{1}$ Este artículo se adscribe al proyecto de investigación $\mathrm{I}+\mathrm{D}$ "Espacios de conocimiento, cultura y agencia femeninas en el mundo Moderno y Contemporáneo (siglos XV-XX), PGC2018-097445-B-C21, subproyecto del Coordinado, "Género, cultura y subjetividad: más allá de las políticas del conocimiento (siglos XV-XX)", Ministerio de Ciencia, Innovación y Universidades.

DOI del artículo:

https://doi.org/10.25267/Cuad investig fondos arch UCA.2020.i2.08 
Si alguien piensa que las palabras «infancia»y «niños» van dirigidas tanto a ellos como a ellas, en el artículo 115 de este mismo Proyecto podrá leer: «Se establecerán escuelas públicas, en que se enseñe á las niñas á leer y escribir, y á las adultas las labores y habilidades propias de su sexo». Con leer y escribir ya iban las niñas servidas, pero las enseñanzas de las labores de «su sexo», al parecer, no les faltaban a ellas.

El Reglamento de las Escuelas Públicas de Instrucción primaria elemental, de 26 de noviembre de 1838, decía que, como no era obligatorio establecer escuelas de niñas, proponía que se estableciesen estas escuelas en los mismos edificios que las de niños, pero en salas separadas, sin que el maestro desatendiera la clase de niños ni un momento, ya que los niños eran el principal objeto de la enseñanza. No obstante, la administración tenía otra propuesta: poner a otra persona al frente de la clase de niñas. Si alguien está pensando que esa otra persona podría ser una maestra, se equivoca. Sería la esposa del maestro, sin exigencia de título. Es más. Si la esposa estuviera ocupada, o no hubiera esposa, la escuela de niñas estaría atendida por la sirvienta del maestro. Si ya la economía de los maestros no estaba para muchas alegrías, ¿cómo debía estar la de las sirvientas? Si las niñas de las clases más desfavorecidas no accedían a la enseñanza por falta de escuelas y porque tenían que trabajar, muchas de ellas como sirvientas, ¿cómo podían atender a una clase de niñas?

Es evidente que la preparación de estas personas no era la preocupación primordial del Gobierno ya que, como decían las propias autoridades, para impartir enseñanza en una escuela de niñas no se necesitaban grandes conocimientos. Como también decía el Reglamento, además de hacer un gran servicio público, los maestros favorecían sus intereses y contribuían a mejorar su suerte, ya que recibían las retribuciones de las alumnas que tenían cierta capacidad económica. Así pues, no quedaba claro si la finalidad principal de la instalación de estas escuelas de niñas era la de procurar su instrucción o la de aliviar la maltrecha economía de los maestros, tan mal pagados y considerados (Sánchez Villanueva 2012, p. 277).

Si las autoridades consideraban que las niñas no necesitaban instrucción, se deduce que tampoco necesitaban maestras que estuvieran bien instruidas, ni siquiera mal instruidas. Sin embargo, las que estaban implicadas, como eran las que querían ser maestras, se procuraban su preparación recurriendo a sus familiares, a sus amistades o a ellas mismas, a su interés y esfuerzo, como fue el caso de Dolores Montero Toribio, que envió esta carta al presidente y vocales de la Junta local de Instrucción Pública.

Dolores Montero Toribio, de diez y seis años de edad, natural y vecina de esta Ciudad, á V.S.S. reverentemente expone: Que siendo alumna de la escuela pública de niñas de Ntra. Sra. de Consolacion ha cursado en dicha escuela los estudios necesarios para poderse examinar de Maestra de Instruccion Primaria Elemental; y faltándole estar en la Normal de Cádiz siquiera dos meses para oir y adaptarse á las esplicaciones de los Profesores de la misma, y careciendo de recursos, no solo para sostenerse en Cádiz sino aun para costear el Título por su estremada pobreza.

Suplica á V.S.S. se interese con el Exmo. Ayuntamiento para que, de los fondos que se destinen para premios de las Escuelas Públicas, se le pudiere adjudicar una cantidad con que sufragar estos gastos. 
Gracia que espera alcanzar de la bondad de V.S.S. cuyas vidas guarde Dios muchos años.

Jerez 10 de Junio de $1875 . \quad$ Dolores Montero Toribio ${ }^{2}$.

Citaremos, por último, los artículos 2, 3 y 4 del Proyecto de Ley de Instrucción Pública, de 9 de diciembre de 1855, por su cercanía a la Ley Moyano, de 1857. Las materias que debían cursar tanto los niños como las niñas eran: doctrina cristiana, lectura, escritura, principios de gramática castellana y reglas de ortografía y principios de aritmética, con el sistema legal de medidas, pesas y monedas. Además de las materias anteriores, los niños recibirán breves nociones de agricultura, de industria o de comercio, al mismo tiempo que las niñas harán «labores propias de su sexo»; los niños estudiarían rudimentos de geografía e historia, al mismo tiempo que las niñas harán «labores propias de su sexo»; los niños trabajarían los principios de geometría con aplicación al dibujo lineal, al mismo tiempo que las niñas harán «labores propias de su sexo»; los niños tendrán nociones elementales de las ciencias físicas y naturales aplicables a los usos comunes de la vida, al mismo tiempo que las niñas harán «labores propias de su sexo».

Después de la lectura de estos ejemplos sacados de la legislación sobre enseñanza, podemos afirmar que las autoridades públicas no se han preocupado de que la enseñanza, en cualquiera de sus niveles, haya estado al alcance de las mujeres en ninguna de las etapas de su vida. No ya en los niveles superiores, como la universidad, o en la segunda enseñanza, como los institutos, ni siquiera en la enseñanza más elemental y primaria. Hubo que esperar a 1857, a la ley Moyano, para que las leyes acercaran los derechos de las niñas a los de los niños.

La sociedad del siglo XIX no dejaba mucho margen para que las mujeres llevaran a cabo otras tareas, otras profesiones, que las apartara de las «labores propias de su sexo». La enseñanza y la enfermería eran dos de esas profesiones que se les permitía a las mujeres. Había otras que, no sólo se les permitía, sino que las mujeres se veían obligadas a realizar porque la sociedad machista se lo imponía: la limpieza, la costura, la cocina, el cuidado de los familiares mayores o enfermos, etc.

Antes del establecimiento de las escuelas de magisterio las personas que pretendían dedicarse a la enseñanza debían superar unas pruebas ante una Junta examinadora, pero la preparación, la adquisición de los conocimientos, debían hacerla con la ayuda de una maestra o un maestro. En el caso de Jerez se tienen noticias del establecimiento de tribunales para examinar a los pretendientes de maestros de primeras letras desde el 24 de mayo de 1534, a partir de una real orden de esa misma fecha que permitía a su ayuntamiento formar tribunales.

Ya en el siglo XIX, la maestra Lucrecia Recio y Orozco abrió una escuela de magisterio en Jerez, en la calle San Miguel, número 1, el 15 de septiembre de 1872. La que tuvo mayor

\footnotetext{
${ }^{2}$ Archivo Municipal de Jerez de la Frontera (AMJF), Archivo Histórico Reservado (AHR), C. 20, n. ${ }^{\circ}$ 18, 1875, doc. f. 11. Dolores Montero consiguió en 1876 el título de grado elemental y en 1877 el título de grado superior: Archivo de la Universidad de Cádiz (AUCA), C-71-21 EN y PLAZA DE PRADO, ALICIA y PASCUAL PASCUAL, MARÍA SOLEDAD 2000, p. 226. En este documento se ha respetado la textualidad, aun en sus faltas, y se ha renunciado a poner el adverbio latino (sic) continuamente. 
número de alumnos fue la Escuela preparatoria para Maestros de Primera Enseñanza de Jerez, ubicada en la Alameda Cristina, número 11, en el ex-convento de San Juan de Dios. Se creó en 1878 y estaba incorporada a la Normal de Cádiz. Era Enrique Martín Morilla el director y María Josefa Lafragüeta y del Rey la directora. Impartían, entre otras asignaturas, Práctica de la enseñanza. Debían permanecer, al menos, seis meses de prácticas, después de los cuales los profesores expedían un certificado que debían presentar en Cádiz capital, en la Normal. Francisca Doblado y Gil, maestra de una escuela pública, tenía un colegio particular donde preparaba a jóvenes para la carrera de magisterio ${ }^{3}$ : Colegio preparatorio para maestras de primera enseñanza de Jerez. Lo estableció en 1881, en la plaza de San Lucas, número 4. Ella era directora y profesora. El Colegio de Señoritas de Santa Teresa de Jesús, situado en la calle Honda, número 17, estaba dirigido por Manuel Medina y Aguilar ${ }^{4}$. Por último, citaremos la Escuela de Magisterio de primera enseñanza para señoritas en Porvenir, 26 principal, bajo la dirección de Antonio Bascón Anguita. El programa que ofrecía se ajustaba al exigido por la Normal de Maestras de Cádiz ${ }^{5}$.

A medida que nos vamos adentrando en la segunda mitad del siglo XIX el número de profesionales de la enseñanza con título conseguido en tribunales o juntas examinadoras disminuye, mientras que aumentan los que acceden a la titulación a través de las escuelas normales. «En 1843 se publicó una real orden para que las corporaciones municipales, a la hora de proveer plazas en las escuelas públicas, en igualdad de circunstancias, dieran preferencia a los procedentes de las escuelas normales, siempre, lógicamente, que estuvieran aprobados y con su correspondiente título» (Sánchez Villanueva 2012, p. 163).

Muchos hijos o hijas tienen como referente laboral a su padre o a su madre. En el caso de los maestros, además, hacen su preparación en la escuela de su progenitor, manteniendo el sistema gremial. Muchas niñas, al tener pocas salidas profesionales por un lado y poder prepararse en la casa de su propia familia, por otro, optaban por el magisterio. Además, la sociedad consideraba que las mujeres no necesitaban estar instruidas, tan sólo dominar las tareas domésticas para llevar bien su casa cuando le llegara el matrimonio, fin que la sociedad tenía reservado a la mujer en general.

La exclusión de las mujeres de las leyes educativas; la limitación para trabajar en otras profesiones que no fueran las consideradas propias del hogar, exceptuando la enseñanza; la prohibición para ejercer las profesiones consideradas propias de hombres; el fomento, cuando no la obligación o imposición, de las llamadas labores del hogar; el hecho de que muchas mujeres vieran en el magisterio un posible puesto de trabajo, un nivel de preparación reconocido oficialmente y un enriquecimiento cultural (Vázquez Domínguez 2019, p. 78), todos estos factores le llevan a matricularse en la Escuela Normal, aunque luego no hagan uso de su título porque no tengan necesidad de ejercer como maestras.

La preparación para superar los exámenes de ingreso en la Escuela Normal la suelen hacer en las escuelas de enseñanza primaria de su ciudad, de su barrio o de sus familiares, porque la familia es otro de los factores que fomenta el interés, el deseo o la vocación por la

\footnotetext{
${ }^{3}$ AMJF, AHR, C. 20, n. ${ }^{\circ} 18,1881$, Actas, f. 21.

${ }^{4}$ El Magisterio Jerezano, 16 de julio de 1883, n. ${ }^{\circ}$ 56, p. 1.

${ }^{5}$ El Guadalete, 8 de septiembre de 1889, p. 3.
} 
enseñanza. No son pocos los casos de familias que al completo o parcialmente se han dedicado a la enseñanza.

Las maestras que han nacido o ejercido en Jerez y que han estudiado en la Escuela Normal de Maestras de Cádiz son 270. Las que son hermanas en esa lista llegan a 50 maestras. Eso quiere decir que suponen una cantidad importante, un 18,5\%. Por otra parte, hay otra cifra que la forman las que están en esa lista y tienen hermanas fuera de ella porque han obtenido su título en otra entidad. Estas llegan a 85, un 31,5 \%, cifra aún más importante. Además, estarían las familias con componentes masculinos docentes. Podemos deducir que la influencia de la familia es un factor importante a tener en cuenta a la hora de decidirse por una actividad laboral.

A continuación damos a conocer varios ejemplos tomados del Jerez del siglo XIX.

\section{LA FAMilia Doblado, EnRíQueZ y FernándeZ DE CASTRo}

Formada por las hermanas Francisca y M. ${ }^{a}$ de los Reyes Doblado y Gil de Hermoso, José M. ${ }^{a}$ Enríquez y Fuentes, marido de Francisca, y los hijos de M. ${ }^{a}$ de los Reyes: Emilia, Julia, Francisco y Leandro Fernández de Castro y Doblado.

Francisca Gil de Hermoso Jarana y Juan José Doblado, panadero, se encontraban en Lebrija cuando les nació una niña el 30 de enero de 1830 recibiendo el nombre de su madre ${ }^{6}$. También recibiría, pero ya el 16 de agosto de 1853, el título de maestra de instrucción primaria elemental, en esta ocasión después de un examen ante la Junta Superior de Sevilla. En ese mismo mes de agosto se celebraron oposiciones para cubrir dos plazas de maestras de las escuelas públicas que iban a establecerse en Jerez. La Comisión superior propuso a Francisca Doblado como maestra interina. Se aceptó la idea de contratarla, aunque la comisión local consideraba que a sus 23 años era muy joven para dirigir una escuela. Sin embargo, tuvo en cuenta que era de moralidad y costumbres excelentes y que con su trabajo se mantenía ella y mantenía a su madre que vivía con ella ${ }^{7}$.

El 15 de febrero de 1854 se celebraron las nuevas oposiciones y Francisca Doblado volvió a presentarse. La Comisión provincial la propuso como maestra propietaria. Fue nombrada el 12 de diciembre de 1854 con un sueldo de 6.000 reales al año, motivo suficiente como para que la familia Doblado se instalara en Jerez. El 10 de enero de 1855, asistió en calidad de directora a la inauguración de la escuela pública de niñas de la plaza de San Lucas, que en 1857 tendría el nombre de Ntra. Sra. de la Concepción ${ }^{8}$. En esta escuela se empleaba un sistema de enseñanza mixto, basado en el mutuo. Estuvo allí como directora desde el 10 de enero de 1855 hasta el 4 de noviembre de 1898 en que se jubiló. Durante esos casi 44 años pasaron por el puesto de ayudanta numerosas maestras de entre las que destacamos a su hermana M. ${ }^{a}$ de los Reyes, que estuvo con ella más de 21 años.

\footnotetext{
${ }^{6}$ AMJF, Padrones de 1857, vol. 220, f. 121 y de 1889, vol. 699, f. 1072.

${ }^{7}$ AMJF, AHR, C. 20, n. ${ }^{\circ} 7$, Junta n. ${ }^{\circ} 6$ de 31 octubre 1853, punto $1 .^{\circ}$.

${ }^{8}$ AMJF, AHR, C. 20, n. ${ }^{\circ} 27$.
} 
Francisca se casó el 31 de diciembre de 1855 con José M. ${ }^{a}$ Enríquez y Fuentes ${ }^{9}$, un jerezano que nació el 19 de marzo de 1834 y que también pertenecía a una familia de docentes. Los hermanos José M. ${ }^{a}$, Manuel M. ${ }^{a}$ y Gertrudis Enríquez y Fuentes eran hijos de Gertrudis y Manuel M. ${ }^{\mathrm{a}}$, maestro, que les enseñaría el oficio, manteniendo esa costumbre gremial.

En junio de 1855 José M. ${ }^{a}$ aprobó unas oposiciones de maestro ayudante, tomando posesión de su plaza el 19 de octubre de 1855 en la escuela pública de la plaza de los Santos, titulada de San Miguel ${ }^{10}$. José M. ${ }^{a}$ Enríquez era una persona con inquietudes y quiso estudiar magisterio en la Escuela Normal Superior de Maestros de Cádiz; así que solicitó permiso en agosto de 1863 para poder empezar el curso el 15 de septiembre de ese año, dejando en su lugar a un sustituto, como era habitual ${ }^{11}$. No dejó pasar el tiempo y, con su flamante título, de nuevo se presentó a otras oposiciones; en esta ocasión para optar a la dirección de la escuela pública de párvulos de San Luis Gonzaga junto con su hermana Gertrudis. Consiguieron la dirección en propiedad el 24 de octubre de 1865 y tomaron posesión el 17 de noviembre de ese mismo año.

Si José M. ${ }^{a}$ Enríquez era activo, no menos lo era Francisca Doblado, pues esta había creado un centro docente, llamado Colegio preparatorio para maestras de primera enseñanza de Jerez. Lo estableció en 1881, anexo a la escuela elemental de niñas de Ntra. Sra. de la Concepción de la que era directora. Las alumnas de esta escuela de magisterio hacían sus prácticas como instructoras en la misma escuela pública de niñas ${ }^{12}$. En 1887 se contabilizaron más de cien alumnas matriculadas, con buenos resultados en los exámenes públicos, confirmados por las brillantes notas obtenidas en la Escuela Normal Superior de Maestras de Cádiz ${ }^{13}$. Cuando alguna de sus alumnas se presentaba en la Escuela Normal para continuar sus estudios, Francisca Doblado le extendía un certificado en estos términos:

La que suscribe maestra de las escuelas públicas de Jerez,

Certifica: que la señorita D. ${ }^{a} \ldots$, alumna de primer año en la carrera del Magisterio, cuyos estudios ha recibido privadamente, ha practicado la enseñanza en la escuela de mi cargo durante el curso académico de ..., en la forma que determinan las disposiciones vigentes.

Y para que pueda hacerlo constar, expido la presente en conformidad á la disposición

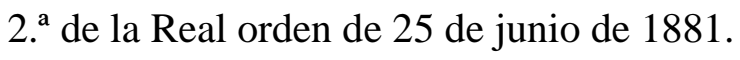

Entre los certificados que llegó a extender, todavía se conservan los de M. ${ }^{\mathrm{a}}$ Antonia Martos y Olavarrieta, Ana M. ${ }^{a}$ Otero y Palacios, M. ${ }^{a}$ de Consolación Pereira y Bara, Ana López Reguera y el de su sobrina Emilia Fernández de Castro y Doblado.

Francisca también participó en la Exposición Regional de Cádiz de 1879 con sus trabajos de taquigrafía (sí, de taquigrafía). Esa intensa actividad le minó su salud. Eso decía el médico en su informe del 10 de agosto de 1856, en el que, por prescripción facultativa, le

${ }^{9}$ AMJF, Padrón de 1857, Vol. 220, f. 121.

${ }^{10}$ AMJF, Leg. 353, Exp. 10360, 1854, f. 85-91.

${ }^{11}$ AMJF, AHR, C. 20, n. ${ }^{\circ} 10,1863$, Actas, doc. 15 de septiembre y C. 20, n. ${ }^{\circ} 11,1864$, Junta local de Instrucción pública, f. 2.

${ }^{12}$ AMJF, AHR, C. 20, n. ${ }^{\circ} 19,1882$, doc. 4 y Actas, f. 1.

${ }^{13}$ AMJF, AHR, C. 20, n. ${ }^{\circ} 19,1882$, Actas, f. 11. 
concedía 25 días para tomar baños de mar, ya que tenía muy delicada su salud a causa del excesivo trabajo; mientras, la clase quedó en manos de su hermana, M. ${ }^{a}$ de los Reyes, que era su ayudante en esos momentos. El 4 de noviembre de 1898 le concedieron la jubilación por edad.

M. ${ }^{a}$ de los Reyes Doblado y Gil de Hermoso, nació en Lebrija el 6 de enero de 1833. A raíz de las oposiciones que ganó su hermana Francisca en 1854 para la plaza de directora de la escuela pública de niñas de Ntra. Sra. de la Concepción, la familia Doblado se mudó a Jere $^{14}$, a la vivienda de la escuela que dirigía esta maestra en la plaza de San Lucas, 5. M. ${ }^{\text {a }}$ de los Reyes se presentó a oposiciones para ayudantas de las mismas escuelas a las que se había presentado su hermana para directora. El domingo 28 de enero de 1855 se celebraron las pruebas. La Comisión local nombró interinamente a las que mejor lo habían hecho: M. ${ }^{a}$ de los Reyes Doblado y M. ${ }^{\text {a }}$ Teresa Naranjo ${ }^{15}$. Seis meses más tarde se volvieron a convocar exámenes a los que se presentaron M. $^{a}$ de los Reyes Doblado y M. ${ }^{a}$ Amalia Naranjo. El Ayuntamiento, el 19 octubre de 1855, nombró a M. ${ }^{a}$ de los Reyes ayudanta en propiedad de la escuela de niñas de la plaza de San Lucas, Ntra. Sra. de Concepción, con el sueldo anual de 3.000 reales. M. ${ }^{a}$ de los Reyes viviría en el mismo domicilio que su hermana Francisca de la que era ayudanta.

Al parecer todos los miembros de esta familia eran inquietos. M. ${ }^{a}$ de los Reyes pidió licencia para asistir durante ocho meses a la Escuela Normal Superior de Maestras de Cádiz. Obtendría el título de maestra de enseñanza elemental con nota de sobresaliente ${ }^{16}$. Al igual que otros miembros de la familia también fue muy inquieta y pasó por numerosas escuelas. En 1895, ya con 62 años, se encontraba en Don Benito, Badajoz, en una escuela pública de niñas ${ }^{17}$.

M. ${ }^{a}$ de los Reyes se casó el 1 de febrero de 1865 con Lorenzo Fernández de Castro ${ }^{18}$, maestro de escuela ${ }^{19}$. Emilia fue la primera de sus hijas y la primera que se dedicó a la enseñanza. Nació el 21 de mayo de 1866, cuando sus padres vivían en la calle Cabezas, número 14, en Jerez. La bautizaron el 23 del mismo mes y año en la iglesia de San Mateo ${ }^{20}$. Después pasaría a vivir con su madre y con su tía Francisca, en la plaza de San Lucas, número $5^{21}$.

La autorización para poder estudiar en la Escuela Normal Superior de Maestras de la Provincia de Cádiz estaba firmada tanto por el padre como por la madre, el 27 de septiembre de 1881. Un día más tarde solicitó hacer el examen de ingreso, solicitud que le fue aceptada, realizando el examen el mismo día con un resultado positivo. Presentó los certificados del Ayuntamiento, del médico y del párroco, que decían de ella que manifestaba buena conducta, que no padecía enfermedad alguna contagiosa y que su conducta moral y religiosa

\footnotetext{
${ }^{14}$ AUCA, C-49-22 EN.

15 AMJF, Leg. 353, Exp. 10359, 1852, f. 172-173 y 176 y Leg. 353, Exp. 10360, 1854, f. 67-69.

${ }^{16}$ AMJF, AHR, C. 20, n. ${ }^{\circ}$ 9, 1860, Documentos vistos en sesiones.

${ }^{17}$ AUCA, C-9-32 EN.

${ }^{18}$ AMJF, Registro Civil. Casados. 1865, f. 14.

${ }^{19}$ AMJF, Registro Civil, Nacidos, Libro 30, 1869, f. 202.

${ }^{20}$ AMJF, Registro Civil, Nacidos, Libro 27, 1866, f. 206.

${ }^{21}$ AMJF, Padrón 1875, T. 466, f. 265 y 1889, Vol. 699, f. 1072-1074, Plaza de San Lucas, 4.
} 
era buena. Había sido alumna de su tía Francisca Doblado en el Colegio de maestras que esta dirigía; y durante el curso académico de 1881-82 realizó en él la asignatura Práctica de la enseñanza. Francisca Doblado le extendió un certificado para que lo presentara en la Escuela Normal de Maestras cuando fuera a estudiar allí.

El 28 de septiembre de 1882 se matriculó de las asignaturas del curso 1882-83 como alumna de estudios privados. Un año más tarde, el 29 de septiembre de 1883, se matriculó de las asignaturas del tercer curso, igualmente como alumna de estudios privados. El 15 de diciembre de 1883 conoció el resultado del examen de reválida para el título de maestra de primera enseñanza elemental, calificada con la nota de Buena en el ejercicio escrito, la de Sobresaliente en el oral, la de Aprobado en el de labores, y la definitiva de Sobresaliente. El 18 de diciembre de 1884 conoció el resultado del examen de reválida para el título de maestra de primera enseñanza superior, calificada con la nota de Buena en el ejercicio escrito, la de Sobresaliente en el oral, la de Sobresaliente en el de labores, y la definitiva de Sobresaliente $^{22}$. El título superior le fue expedido en Madrid por el Ministro de Fomento el 18 de julio de $1885^{23}$.

Entró como maestra ayudanta de la escuela pública de párvulos de San Luis Gonzaga en 1895 y estuvo hasta el 26 de septiembre de 1900, momento en que renunció ${ }^{24}$. En 1898 hizo de directora accidental por fallecimiento del titular, José M. ${ }^{a}$ Enríquez y Fuentes que falleció el 23 de agosto $^{25}$.

Julia, hija también de M. ${ }^{a}$ de los Reyes y de Lorenzo Fernández de Castro, nació en Jerez, el 4 de abril de 1869 y en la calle Moral, número 5. A ella la bautizaron en la iglesia de San Lucas cuatro días después de nacer, también tuvo como domicilio la calle Cabezas ${ }^{26}$.

El 26 de septiembre de 1883 solicitó permiso para hacer el examen de ingreso en la Escuela Normal Superior de Maestras de Cádiz. La autorización paternal la presentó el 20 de septiembre de 1883. El certificado del Ayuntamiento nos dice que era de buena conducta; por el médico Cayetano Pérez y Fuentes sabemos que estaba vacunada, «que no padecía afección contagiosa», y el cura de San Mateo certificaba que era una «persona de buena conducta moral y religiosa ${ }^{27}$, según le constaba del conocimiento que tenía de la misma y de su familia. Las prácticas de enseñanza las realizó durante el curso 1884-85 en la escuela pública de niñas de Ntra. Sra. de la Concepción dirigida por su tía Francisca Doblado ${ }^{28}$.

Superó los cursos correspondientes de magisterio, obteniendo el título de maestra de primera enseñanza elemental el 4 de diciembre de 1885, después de superar la reválida. El título del nivel superior lo consiguió el 29 de octubre de 1886, tanto en un caso como en otro el resultado fue de sobresaliente ${ }^{29}$. En 1886 Julia Fernández de Castro estampaba su firma en el impreso de solicitud de matrícula de Eulalia Fernández y Ducha ${ }^{30}$.

\footnotetext{
${ }^{22}$ AUCA, C-52-8 EN.

${ }^{23}$ Archivo General de la Administración, AGA, 31/18047, n. ${ }^{\circ}$ 28, Emilia Fernández de Castro Doblado.

${ }^{24}$ AMJF, AHR, C. 20, n. ${ }^{\circ}$ 22, 1900, Actas y doc. de la Junta Local de primera enseñanza, Acta 6, punto 5.

${ }^{25}$ AMJF, AHR, C. 20, n. ${ }^{\circ} 21,1898$, Actas, sesión 3, punto 5.

${ }^{26}$ AMJF, Padrón 1889, Vol. 699, f. 1072-1074, Plaza de San Lucas, 4.

27 AUCA, C-52-9 EN.

${ }^{28}$ AUCA, C-115-1 EN.

${ }^{29}$ AUCA, C-52-9 EN.
} 
Estuvo como maestra ayudanta interina en la escuela pública de niñas de Ntra. Sra. de Consolación desde el 13 de diciembre de 1886 hasta el 30 de marzo de 1887, en que tomó posesión como ayudanta accidental en la escuela pública de niñas de Ntra. Sra. del Rosario. Allí estuvo hasta el 29 de abril de $1887^{31}$.

Francisco Fernández de Castro y Doblado, nació en Alcalá de los Gazules, en 1874. En septiembre de 1886 solicitó el ingreso en la Escuela Normal de Maestros de la Provincia de Cádiz, su madre le firmó la autorización necesaria ${ }^{32}$. Leandro Fernández de Castro y Doblado, como su hermano, también nació en Alcalá de los Gazules y también se dedicó a la docencia. Comenzó sus estudios de magisterio en septiembre de 1895, con el ingreso en la Escuela Normal Superior de Cádiz.

\section{LA FAMILIA NARANJO}

Cuatro hermanas, con sus títulos de magisterio, forman esta familia: M. ${ }^{a}$ del Carmen, M. ${ }^{a}$ Teresa, M. ${ }^{a}$ Aurora y M. ${ }^{a}$ Amalia Naranjo y Díaz Peña. Tuvieron muchas cosas en común: por supuesto, las propias del hecho de ser hermanas; también la de dedicarse a la docencia y, yo añadiría otra coincidencia, la de tener una salud delicada y ser buenas personas, de buena condición.

M. ${ }^{a}$ Amalia nació en Cádiz el 11 de febrero de 1828 y se bautizó tres días más tarde en la Catedral. Por orden de S.M. la reina, el Ministro de Fomento le expidió el título de maestra de Instrucción primaria elemental en Madrid el 7 de noviembre de 1856. En nombre del Ministro firmó el Director General, José de Posada Herrera ${ }^{33}$.

Se presentó al concurso oposición para maestras ayudantas en 1855, en la escuela de la Victoria. Las plazas las consiguieron M. ${ }^{\mathrm{a}}$ de los Reyes Doblado y M. ${ }^{\mathrm{a}}$ Amalia Naranjo ${ }^{34}$, ésta última comenzó el 19 de octubre de 1855 como maestra ayudanta de la escuela pública de niñas de la calle Antona de Dios, 16, llamada en 1857 de Ntra. Sra. del Rosario, de la que su hermana M. ${ }^{\text {a }}$ Aurora era la maestra directora.

En Antona de Dios vivían las cuatro hermanas y la madre. En una vivienda estaba M. ${ }^{a}$ del Carmen Naranjo y Díaz Peña, viuda a sus 47 años, y sus cuatro hijos. En otra estaban las tres hermanas solteras, M. ${ }^{\mathrm{a}}$ Teresa, M. ${ }^{\mathrm{a}}$ Aurora y M. ${ }^{\mathrm{a}}$ Amalia, con la madre, Josefa. El resto del edificio estaba ocupado por tres familias más y por la escuela pública de niñas ${ }^{35}$.

M. ${ }^{a}$ Amalia sufrió las viruelas en 1856 y, para evitar el contagio de las educandas, se suspendieron las clases durante unos días ${ }^{36}$. En esta escuela estuvo hasta el 30 de septiembre de 1857 en que renunció porque su estado de salud no le permitía estar al nivel que ella deseaba.

\footnotetext{
${ }^{30}$ AUCA, C-52-12 EN.

${ }^{31}$ AMJF, AHR, C. 20, n. ${ }^{\circ}$ 19, 1887, Junta de Primera Enseñanza. Documentos y Actas, doc. 20 y 23.

${ }^{32}$ AUCA, C-9-31 EN.

${ }^{33}$ AUCA, C-74-9 EN.

${ }^{34}$ AMJF, Leg. 353, Exp. 10359, 1852, f. 172-173 y 176 y Leg. 353, Exp. 10360, 1854, f. 67-69.

35 AMJF, Padrón 1857, T. 217.

${ }^{36}$ AMJF, AHR, C. 20, n. ${ }^{\circ} 8,1856$, Cuaderno de actas, f. 63.
} 
M. ${ }^{a}$ Aurora nació en Cádiz en 1829. La segunda de las hermanas Naranjo y Díaz Peña fue nombrada el 12 de diciembre de 1854 maestra directora en propiedad de la escuela pública de niñas de la calle Antona de Dios, 16, conocida poco después por Ntra. Sra. del Rosario. Tomó posesión de su cargo el 10 de enero de 1855, día de la inauguración de la escuela. Su hermana M. ${ }^{a}$ Amalia fue durante algo más de dos años ayudanta suya y, a veces, su otra hermana, M. ${ }^{\text {a }}$ Teresa.

El 28 de julio de 1857 M. a Aurora Naranjo pidió licencia porque no se encontraba muy bien. Como el tratamiento aconsejado con frecuencia en aquella época para restablecer la salud era el de tomar aires de mar, también se lo recomendaron a ella, así que, el 3 de agosto, le concedieron un mes de permiso ${ }^{37}$. M. ${ }^{a}$ Aurora renunció a su plaza en la escuela pública de niñas de Jerez el 12 de octubre de 1857, porque fue elegida profesora interina de labores y rectora del Seminario de maestras de la Escuela Normal Superior de Cádiz. Allí estuvo hasta el 7 de marzo de 1859 en que dimitió por enfermedad: «No permitiéndome el estado delicado de mi salud continuar en el desempeño del destino de Rectora» ${ }^{38}$. Murió poco después, el 20 de mayo de ese mismo año.

M. ${ }^{a}$ Teresa nació en Cádiz el 7 de marzo de 1824 y al día siguiente se bautizó en la parroquia de San Lorenzo. Estuvo seis meses de maestra ayudanta interina en la escuela pública de niñas de la calle Antona de Dios, 16, plaza que obtuvo su hermana M. ${ }^{a}$ Amalia de manera definitiva ${ }^{39}$. En mayo de 1857 la Junta local de Instrucción pública reflejaba en sus actas que M. ${ }^{\text {a }}$ Teresa pensaba entrar como monja en el Beaterio del Santísimo Sacramento ${ }^{40}$. Si lo hizo no duró mucho tiempo pues fue nombrada maestra ayudanta de la escuela pública de niñas de Ntra. Sra. de Consolación el 31 de agosto de 1865, tomando posesión el 15 de septiembre de ese mismo año. A los pocos meses, en noviembre, padeció una entero colitis crónica agudizada que le hizo guardar cama en los momentos en que la enfermedad estaba en su fase más aguda y el médico, Vitalio Coloma, le recetó reposo durante quince o veinte días, al menos, para su completo restablecimiento ${ }^{41}$. Dos años más tarde, el 13 de junio de 1867, el doctor Francisco Revueltas y Montel le prescribió tomar baños de mar, en este caso, alternados con baños de agua dulce, durante los meses de julio y agosto, debido a los padecimientos crónicos que de continuo la molestan; antes de que le llegara la licencia ya se tomó la baja debido a su grave enfermedad. Además, tuvo que pagar de su bolsillo 4 reales diarios a dos jóvenes para que la sustituyeran. A pesar de todo ello, la Junta provincial pidió un informe. No sé si llegó a presentarlo pero un año más tarde renunció a su cargo de auxiliar por «los constantes catarros, que me afectan asistir, cual es mi deber á la clase de Ntra. Sra. del Rosario» ${ }^{42}$.

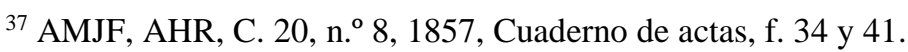

38 AUCA, C-585 EN; AMJF, AHR, C. 20, n. ${ }^{\circ}$ 8, 1857, Documentos, sesión 24, punto 3 y PlAZA DE PRADO, Alicia y Pascual Pascual, María Soledad 2000, pp. 114-115.

${ }^{39}$ AMJF, Leg. 353, Exp. 10359, 1852, f. 172-173 y 176 y Leg. 353, Exp. 10360, 1854, f. 67-69.

${ }^{40}$ AMJF, AHR, C. 20, n. ${ }^{\circ}$ 8, 1857, Actas de la Junta local de instrucción pública, sesión 17, 15 de mayo, punto 3. ${ }^{\circ}$, f. 27 y Leg. 354, Exp. 10364, 1856.

${ }^{41}$ AMJF, AHR, C. 20, n. ${ }^{\circ} 12$, Documentos despachados por la Presidencia, n. ${ }^{\circ} 123$ y 124.

${ }^{42}$ AMJF, AHR, C. 20, n. ${ }^{\circ}$ 14, 1867, Juntas, f. 38, 46 y 50 y Documentos, sesión 7, punto 8 y C. 20, n. ${ }^{\circ} 14$, 1868, Documentos, sesión 5, punto 4.
} 
Los problemas que tenía M. ${ }^{a}$ Teresa de asistencia por enfermedad y de disidencia con la directora de Ntra. Sra. de Consolación hizo que la Junta local la cambiara, el 14 de diciembre de 1867, con la ayudanta de la escuela de Ntra. Sra. del Rosario, M. ${ }^{a}$ de los Reyes Doblado ${ }^{43}$. Estuvo en esta escuela con la directora Antonia del Castillo Iglesias, pero como la enfermedad persistiera M. ${ }^{a}$ Teresa Naranjo renunció a su plaza de ayudanta el 1 de junio de 1868 , renuncia que le fue aceptada por la Junta local ${ }^{44}$.

En julio de 1868 solicitó la apertura de una escuela particular en la calle Santa María, 10, que tendría el nombre de Santa Teresa de Jesús ${ }^{45}$. Pero no abandonó definitivamente la idea de volver a la enseñanza pública ya que le encargó a su sobrina Aurora González que pidiera un certificado de la ayudantía que consiguió en 1855 con la finalidad de incluirlo en el expediente de solicitud de una escuela pública ${ }^{46}$.

\section{M. ${ }^{a}$ Del Rosario y M. ${ }^{a}$ CeCilia García y SÁNCHeZ}

El ejemplo que traigo ahora es el de dos hermanas que tienen poca diferencia de edad. En este caso tan sólo un año de diferencia. M. ${ }^{a}$ Cecilia nació en Jerez el 9 de abril de 1882. Sus padres, M. ${ }^{a}$ del Rosario y Enrique, la bautizaron el 16 de ese mismo mes en la parroquia de Santiago; vivían entonces en la calle Porvera, 29. La otra era M. ${ }^{a}$ del Rosario. Aunque había un año de diferencia en sus edades sus padres decidieron que las dos estudiaran juntas, algo habitual en aquella época. Las dos solicitaron al mismo tiempo, el 19 de septiembre de 1893, el ingreso en la Escuela Normal Superior de Maestra de la Provincia de Cádiz, y se examinaron con éxito dos días después. M. ${ }^{a}$ Cecilia llevó a cabo los ejercicios de reválida para obtener el título de maestra de primera enseñanza elemental entre el 27 y 28 de septiembre de 1898, con buenos resultados, igual que su hermana ${ }^{47}$.

M. ${ }^{a}$ del Rosario, hermana mayor de M. ${ }^{a}$ Cecilia, nació en Jerez el 14 de febrero de 1881. Sus padres la bautizaron el 19 de ese mismo mes en la parroquia de San Miguel. M. ${ }^{\text {a del }}$ Rosario tuvo que esperar un año para solicitar el ingreso en la Escuela Normal Superior de Maestra de la Provincia de Cádiz para hacerlo el 19 de septiembre de 1893, al mismo tiempo que su hermana. Sus padres consideraban que tenían que hacer los estudios al mismo tiempo. Los ejercicios de reválida para obtener el título de maestra de primera enseñanza elemental también los realizó entre el 27 y 28 de septiembre de 1898 con buenos resultados ${ }^{48}$.

\section{REFERENCIAS BIBLIOGRÁFICAS:}

Plaza de Prado, Alicia y Pascual Pascual, María Soledad, 2000, Los archivos de las escuelas normales de Cádiz. Siglo XIX, Cádiz, Servicio de Publicaciones de la Universidad de Cádiz.

\footnotetext{
${ }^{43}$ AMJF, AHR, C. 20, n. ${ }^{\circ}$ 14, 1867, Juntas, f. 58 v y Documentos, sesión 10, punto 5, 14 de diciembre.

${ }^{44}$ AMJF, AHR, C. 20, n. ${ }^{\circ}$ 14, 1868, Junta N. ${ }^{\circ}$ 5, 2 de junio de 1868, f. 18.

${ }^{45}$ AMJF, AHR, C. 20, n. ${ }^{\circ}$ 15, 1868, Junta local de Instrucción primaria, Acuerdos, f. 6.

${ }^{46}$ AMJF, Leg. 353, Exp. 10360, f. 65-66.

${ }^{47}$ AUCA, C-57-11 EN.

48 AUCA, C-57-12 EN.
} 
SÁNCHEZ VILlANUEVA, JUAN LuIS. 2012, El sistema educativo liberal de Jerez: los primeros pasos en la escuela pública, Cádiz, Servicio de Publicaciones de la Universidad de Cádiz.

VÁZqueZ DomíngueZ, CARMEN, 2019. «Nacimiento de las Escuelas Normales gaditanas: un análisis de las matrículas que evidencian su repercusión», Cuadernos de Investigación de Fondos del Archivo UCA, n. ${ }^{\circ} 1$ (2019), pp. 76-87. Disponible en: https://revistas.uca.es/index.php/cifa/article/view/5013/5363. 